\title{
Solar-like oscillation amplitudes and line-widths as a probe for turbulent convection in stars
}

\author{
R. Samadi ${ }^{1}$, K. Belkacem ${ }^{1}$, M.-J. Goupil ${ }^{1}$, F. Kupka ${ }^{2}$ \\ and M.-A. Dupret ${ }^{1}$ \\ ${ }^{1}$ Observatoire de Paris, LESIA, Meudon, France; email: reza.samadi@obspm.fr \\ ${ }^{2}$ Max-Planck-Institute for Astrophysics, Garching, Germany.
}

\begin{abstract}
Excitation of solar-like oscillations is attributed to turbulent convection and takes place at the upper-most part of the outer convective zones. Amplitudes of these oscillations depend on the efficiency of the excitation processes as well as on the properties of turbulent convection. We present past and recent improvements on the modeling of those processes. We show how the mode amplitudes and mode line-widths can bring information about the turbulence in the specific cases of the Sun and $\alpha$ Cen A.
\end{abstract}

Keywords. Turbulence, convection, Sun: oscillations, stars: oscillations (including pulsations), stars: Alpha Cen A

\section{Introduction}

Solar-like oscillations have now been detected in a dozen main-sequence stars as well as in some red giant stars (see the recent review by Bedding \& Kjeldsen 2006). Excitation of such oscillations is ensured by turbulent convection at the upper-most part of the convective zones. From the measurement of the mode amplitude and line-width, it is possible to infer the power supplied to the mode by turbulent convection. As highlighted in the present proceedings, this in turn permits to probe the turbulent convection in stars.

Such measurements have been available for the Sun for several years and give the possibility to test the various proposed theoretical models of mode excitation (e.g. Goldreich \& Keeley 1977; Balmforth 1992; Samadi \& Goupil 2001; Chaplin et al. 2005). We consider here the model of Samadi \& Goupil (2001, SG01 hereafter) with the improvements proposed by Belkacem et al. (2006b, B06b hereafter)

The theoretical model of SG01 requires a prescription for the time-correlation between the turbulent elements. On the basis of a 3D simulation of the upper part of the solar convective zone, Samadi et al. (2003a, SNS03 hereafter) have shown that the eddy timecorrelation, at large scale, is better fitted by a Lorentzian function (LF hereafter) than a Gaussian function (GF hereafter). Furthermore, excitation rates of solar p modes computed on the basis of the model of SG01 with a LF better agree with the excitation rates inferred from the helioseismic observations by Chaplin et al. (1998) than when using a GF. The open question is whether or not this result remains valid for other stars.

The theoretical model of stochastic excitation requires a prescription for the fourth order moments (FOM hereafter) involving the entropy fluctuations and the turbulent velocity. SG01 assume the quasi-normal approximation (QNA) which consists in splitting the FOM into the product of two second-order moments (SOM hereafter). In the solar convective zone, this simple closure model is however significantly biased as verified by 
the recent studies performed by Belkacem et al. (2006a, B06a hereafter) and Kupka \& Robinson (2007, and this volume). Furthermore, as shown in B06b, the skew introduced by the QNA leads to an under-estimation of the solar p mode excitation rates.

Closure models more sophisticated than the QNA can be used. Among those, the socalled two-scale mass flux model (TFM hereafter) improved by Gryanik \& Hartmann (2002) takes the asymmetries in the medium into account but is only applicable for quasi-laminar flows. B06a have generalized Gryanik \& Hartmann (2002)'s approach by taking the turbulent properties of the medium into account. In order to apply this model in the solar case, they have introduced the plumes dynamics following Rieutord \& Zahn (1995). Furthermore, as shown in B06b, the calculations based on this new closure model increases the contribution of the Reynolds stress contribution to the excitation rates of the solar modes. When the additional contribution due to the entropy fluctuations is included, the new theoretical calculations fit rather well the maximum in the solar mode excitation rates derived by Baudin et al. (2005). All the results by B06b are summarized and discussed in the present proceedings (see also Belkacem et al., this volume).

Apart from the Sun, mode amplitudes and mode line-widths have been derived for few stars. Among those stars, $\alpha$ Cen Aprovides the best available data. Bedding et al. (2004) have derived the mode amplitudes and the mode line-widths for $\alpha$ Cen A. These mode line-widths have been recently updated by Kjeldsen et al. (2005). More recently, Fletcher et al. (2006) have derived the mode line-widths from the WIRE observations of $\alpha$ Cen A(Schou \& Buzasi 2001). From those new measurements we have updated the work performed in Samadi et al. (2004) by deriving new constraints on the rate at which energy is supplied by unit time to the p modes of $\alpha$ Cen A. These constraints are compared here with new theoretical calculations performed in the manner of B06b.

This report is organized as follows: in $\S 2$ we briefly recall the model of SG01 with the modifications proposed by B06b. We also present the different ways the eddy-time correlation is described and the different closure models investigated by B06a. Applications of this improved excitation model are shown in $\S 3$ to the case of the Sun $(\S 3.1)$ and of $\alpha$ Cen $\mathrm{A}(\S 3.2)$. Finally $\S 4$ and $\S 5$ are dedicated to conclusion and discussion respectively.

\section{Modeling the stochastic excitation}

The model of stochastic excitation we consider basically is that of SG01 with the improvements proposed by B06b: two terms are expected to drive stochastically the p modes: one term corresponds to the Reynolds stress, the second one is an entropy source term that corresponds to the advection of entropy fluctuations by turbulent motions. The energy supply per unit time into each mode is given by SG01:

$$
P=\frac{1}{8 I}\left(C_{R}^{2}+C_{S}^{2}\right)
$$

where $C_{R}^{2}$ and $C_{S}^{2}$ are the turbulent Reynolds stress and entropy contributions respectively. Their expressions are for radial modes:

$$
\begin{array}{r}
C_{R}^{2}=\int_{M} d m \rho_{0} f_{r} \int_{-\infty}^{+\infty} d \tau e^{-i \omega_{0} \tau} \int d^{3} r\left\langle w_{1}^{2} w_{2}^{2}\right\rangle[\vec{r}, \tau] \\
C_{S}^{2}=\int_{M} d m \alpha_{s}^{2} \frac{g_{r}}{\rho_{0}} \int_{-\infty}^{+\infty} d \tau e^{-i \omega_{0} \tau} \int d^{3} r\left\langle\left(w s_{t}\right)_{1}\left(w s_{t}\right)_{2}\right\rangle[\vec{r}, \tau]
\end{array}
$$

where $\rho_{0}$ is the mean density, $w$ the vertical component of the velocity, $s_{t}$ the entropy fluctuation due to turbulence, $\alpha_{s} \equiv \partial(p / \partial s)_{\rho}, m$ the mass enclosed in a sphere with radius 
$r, I$ the mode inertia, $f_{r}\left(\xi_{r}, m\right) \equiv\left(\frac{\partial \xi_{r}}{\partial r}\right)^{2}, \xi_{r}$ the radial component of the displacement eigenfunction, $g_{r}$ is a function that involves the first and second derivatives of $\xi_{r}$ (see Eq. (9) of Samadi et al. 2003b), finally $\tau$ and $r$ are variables related to the time and space correlation lengths respectively.

Quantities labeled with 1 and 2 are taken at the spatial and temporal positions $\left[\vec{x}_{0}-\right.$ $\left.\frac{\vec{r}}{2},-\frac{\tau}{2}\right]$ and $\left[\vec{x}_{0}+\frac{\vec{r}}{2}, \frac{\tau}{2}\right]$ respectively: they correspond to two-point correlation products. The fourth-order two-point correlation product involving the velocity, $\left\langle w_{1}^{2} w_{2}^{2}\right\rangle$, is related to the fourth-order one-point correlation product, $\left\langle w_{1}^{4}\right\rangle$, as summarized in $\S 2.2$. We point out that the fourth-order one-point correlation product, $\left\langle w_{1}^{4}\right\rangle$, corresponds to a FOM in the usual sense of Reynolds averages. In turn, the one-point correlation product, i.e. the FOM, is modeled as described in $\S 2.1$.

\subsection{Closure models}

The QNA (see Lesieur 1997, Chap VII-2) allows us to calculate rather easily the FOM of $w$ in terms of a product of the SOM, that is:

$$
\left\langle w^{4}\right\rangle=3\left\langle w^{2}\right\rangle^{2}
$$

The QNA is strictly valid for normally distributed fluctuating quantities with zero mean. However, the upper-most part of the convection zone being a turbulent convective system composed of essentially two flows, the probability distribution function of the fluctuations of the vertical velocity and temperature do not follow a gaussian law (see e.g. Lesieur 1997, B06a). Hence the use of the QNA is not really valid. This was verified by B06a and Kupka \& Robinson (2007) with the help of 3D simulations of the outer layer of the Sun: Figure 2.1 shows the ratio between the FOM $\left\langle w^{4}\right\rangle$ derived from a 3D simulation as detailled in $\mathrm{B} 06 \mathrm{a}$ and the FOM derived from different closure models. As shown in Figure 2.1, the use of the QNA under estimates, in the quasi-adiabatic region, by $\approx 50 \%$ the FOM of the velocity derived from the $3 \mathrm{D}$ simulation.

A more sophisticated closure model, the mass flux model (Abdella \& McFarlane 1997, MFM hereafter), takes the effects of updrafts and downdrafts on the correlation products into account. Indeed, the presence of two flows introduces a skew when averaging the fluctuating quantities, since averages of fluctuating quantites over each individual flows differ from averages over the total flow. The basic idea of the MFM is to split any averaged turbulent quantity $\phi$ into two parts, one associated with the updrafts and the other with the downdrafts. Gryanik \& Hartmann (2002) have proposed their so-called two-scale mass-flux model (TFM hereafter) which extends the MFM by accounting for the fact that hot drafts (or cold ones, resp.) do not necessarily coincide with the updrafts (downdrafts, resp.). In order to close the third order moments and the FOMs, Gryanik \& Hartmann (2002) adopt the simplifying approximation that $\left\langle\phi^{n}\right\rangle=\langle\phi\rangle^{n}$ where $\langle$. denotes ensemble spatial (in the horizontal plane) and time averages. Hence, this approximation consists in neglecting the turbulence within the flow. Finally, Gryanik \& Hartmann (2002) derive analytical expressions for the third-order moments and FOMs involving the temperature and the velocity. For $\left\langle w^{4}\right\rangle$, Gryanik \& Hartmann (2002) obtained the following expression:

$$
\left\langle w^{4}\right\rangle=\left(1+S_{w}^{2}\right)\left\langle w^{2}\right\rangle^{2}
$$

with the skewness, $S_{w}$, given by:

$$
S_{w} \equiv \frac{\left\langle w^{3}\right\rangle}{\langle w\rangle^{3 / 2}}=\frac{1-2 a}{\sqrt{a(1-a)}}
$$




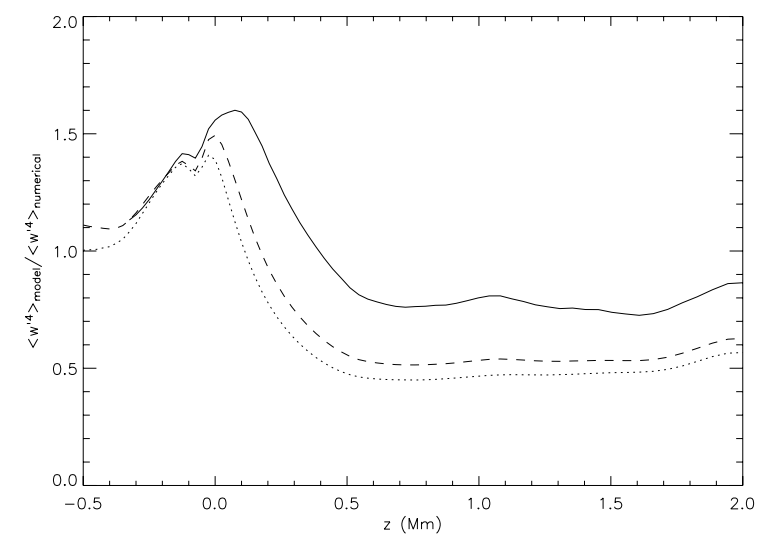

Figure 1. Fourth-order moment (FOM) of the velocity, $\left\langle w^{4}\right\rangle$, as a function of depth $z$, normalized to the FOM derived from the 3D simulation. In solid lines the FOM calculated according to the CMP model, i.e. with the help of Eq. 2.7 and with the skewness, $S_{w}$, given by Eq. 2.8, the dashed line is computed according to the Gryanik \& Hartmann (2002)'s TFM given by Eq. 2.7 with $S_{w}$ given by with Eq. 2.6 and finally the dotted line is the QNA, Eq. 2.4.

where $a$ is the mean fractional area occupied by the updrafts in the horizontal plane. In the QNA limit, i.e. when the random quantities are distributed according to a normal distribution with zero mean, we have necessarily $S_{w}=0$. Hence, in the QNA limit, Eq. 2.5 does not match Eq. 2.4. Gryanik \& Hartmann (2002) proposed to modify Eq. 2.5 as follows (see Kupka \& Robinson 2007 for a discussion of this step):

$$
\left\langle w^{4}\right\rangle=3\left(1+\frac{1}{3} S_{w}^{2}\right)\left\langle w^{2}\right\rangle^{2} .
$$

Figure 2.1 shows that the FOM based on Eq. 2.7 with $S_{w}$ given by Eq. 2.6, results in a negligible improvement with respect to the QNA. However, when $S_{w}$ is derived directly from the 3D simulation and plugged into Eq. 2.7, Eq. 2.7 is a very good evaluation of the FOM derived from a 3D simuation of the outer layer of the Sun as verfied by B06a and Kupka \& Robinson (2007).

B06a have generalized the TFM by taking both the skew introduced by the presence of two flows and the effects of turbulence inside each flow into account. Accordingly, they have derived a more accurate expression for $S_{w}$, that is:

$$
S_{w}=\frac{1}{\left\langle w^{2}\right\rangle^{3 / 2}} a\left((1-a)(1-5 a) \delta w^{2}-3\left\langle w^{2}\right\rangle\right) \delta w
$$

where $\delta w$ is the difference between the average velocity of the up- and the downdrafts and $\left\langle w^{2}\right\rangle$ is the SOM. Both $a$ and $\left\langle w^{2}\right\rangle$ are supposed to be known. Finally, in order to close the system, $\delta w$ needs to be modeled. This is undertaken in B06a by using the plume model of Rieutord \& Zahn (1995). More details can be found in B06a or in Belkacem et al. (2006c).

\subsection{Two-point and Eddy-time correlations}

As seen in Eq. 2.2 and Eq. 2.3, the model of stochastic excitation relies on a prescription for the two point correlation products involving the velocity (Eq. 2.2 and Eq. 2.3) and the entropy (Eq. 2.3). B06b proposed to relate the two-point correlation products involving the velocity to the one-point correlation with the help of QNA as follows:

$$
\left\langle w_{1}^{2} w_{2}^{2}\right\rangle=\frac{\left\langle w^{4}\right\rangle}{\left\langle w^{4}\right\rangle_{\mathrm{QNA}}}\left\langle w_{1}^{2} w_{2}^{2}\right\rangle_{\mathrm{QNA}}
$$

where $\left\langle w^{4}\right\rangle /\left\langle w^{4}\right\rangle_{\mathrm{QNA}}$ is the ratio betwen the FOM of $w$ and the one given by the QNA (Eq. 2.4), $\left\langle w_{1}^{2} w_{2}^{2}\right\rangle_{\mathrm{QNA}}$ is the two-point correlation product given according to the QNA 
(see Lesieur 1997, Chap VII-2), that is:

$$
\left\langle w_{1}^{2} w_{2}^{2}\right\rangle_{\mathrm{QNA}}=2\left\langle w_{1} w_{2}\right\rangle^{2}+\left\langle w_{1}^{2}\right\rangle\left\langle w_{2}^{2}\right\rangle
$$

Note that the second term in Eq. 2.10 does not contribute to the excitation. The FOM in Eq. 2.9 is here computed according to the closure models presented in $\S 2.1$. When the CMP is adopted, from Eqs. 2.9, Eq. 2.7 and Eq. 2.4 one derives the following expression for $\left\langle w_{1}^{2} w_{2}^{2}\right\rangle$ (Eq. 7 of B06b):

$$
\left\langle w_{1}^{2} w_{2}^{2}\right\rangle=\left(1+\frac{1}{3} S_{w}^{2}\right)\left\langle w_{1}^{2} w_{2}^{2}\right\rangle_{\mathrm{QNA}}
$$

where $\left\langle w_{1}^{2} w_{2}^{2}\right\rangle_{\mathrm{QNA}}$ is given by Eq. 2.10.

SG01 introduce $\phi_{i j}(\vec{k}, \omega)$ the time and space Fourier transform of $\left\langle w_{1} w_{2}\right\rangle_{\mathrm{QNA}}$ (the first term in Eq. 2.10). SG01 modeled $\phi_{i j}(\vec{k}, \omega)$ as follows:

$$
\phi_{i j}(\vec{k}, \omega)=\frac{E(k, \omega)}{4 \pi k^{2}}\left(\delta_{i j}-\frac{k_{i} k_{j}}{k^{2}}\right) \text { with } E(k, \omega)=E(k) \chi_{k}(\omega)
$$

where $E(k, \omega)$ is the turbulent kinetic energy spectrum, $\delta_{i j}$ the Kroenecker symbol, $E(k)$ the mean kinetic energy spectrum and $\chi_{k}(\omega)$ the eddy-time correlation function. Several eddy-time correlation functions have been investigated by SNS03. Among those, we compare here the Gaussian function (GF hereafter) and the Lorentzian function (LF hereafter). Finally from Eqs. 2.9-2.12, B06b derive the CMP version of Eq. 2.2 (that is Eq. 10 of B06b). Note that as in SG01 and B06b, the entropy source term (Eq. 2.3) is still modelled on the basis of the QNA.

\section{Results}

\subsection{The Sun}

The calculations of the excitation of the solar modes is performed as detailed in B06b: the radial eigenfunctions, $\xi_{\mathrm{r}}$, eigenfrequencies, $\omega_{0}$, and the mode inertia, $I$, are those used by Samadi et al. (2003b) and originate from an 1D solar model built according to Gough's (1977) non-local formulation of the mixing-length theory. The spatial and time averaged quantities are obtained from a 3D simulation of the solar surface computed with Stein \& Nordlund (1998)'s code and with a grid of $125 \times 125 \times 82$. The total kinetic energy, $E(k)$, in Eq. 2.12 and its depth dependence are obtained directly from the 3D simulation. Finally, quantities related to the convection (the SOM of $w$ and $s_{t}$, and $a$ ) and thermodynamic quantities (the mean pressure $p_{0}, \rho_{0}$ and $\alpha_{s}$ ) are also obtained from the 3D simulation. The results of the calculations of the mode excitation rates (Eq.2.1) are shown for the Sun in Figure 3.2, top panel.

\section{2. $\alpha$ Cen $A$}

Calculations of mode excitation rates are performed for $\alpha$ Cen Ain a similar way as for the Sun: the simulation has a grid of $125 \times 125 \times 82$, an effective temperature of $T_{\text {eff }}=5805 \pm 20 \mathrm{~K}$ and a gravity of $\log g=4.305$ in agreement with the constraints derived from interferometry (see Miglio \& Montalbán 2005). We compute a 1D model with the CESAM code assuming standard physics and the same $T_{\text {eff }}$ and $\log g$ as for the simulation. Finally, $\xi_{\mathrm{r}}, \omega_{0}$ and $I$ are computed with an adiabatic code from the 1D model. The results of the calculations of the mode excitation rates are shown for $\alpha$ Cen Ain Figure 3.2, bottom panel. From the observed amplitude spectrum derived by Kjeldsen et al. (2005) and the different mode line-width measurements, we derive the constraints 


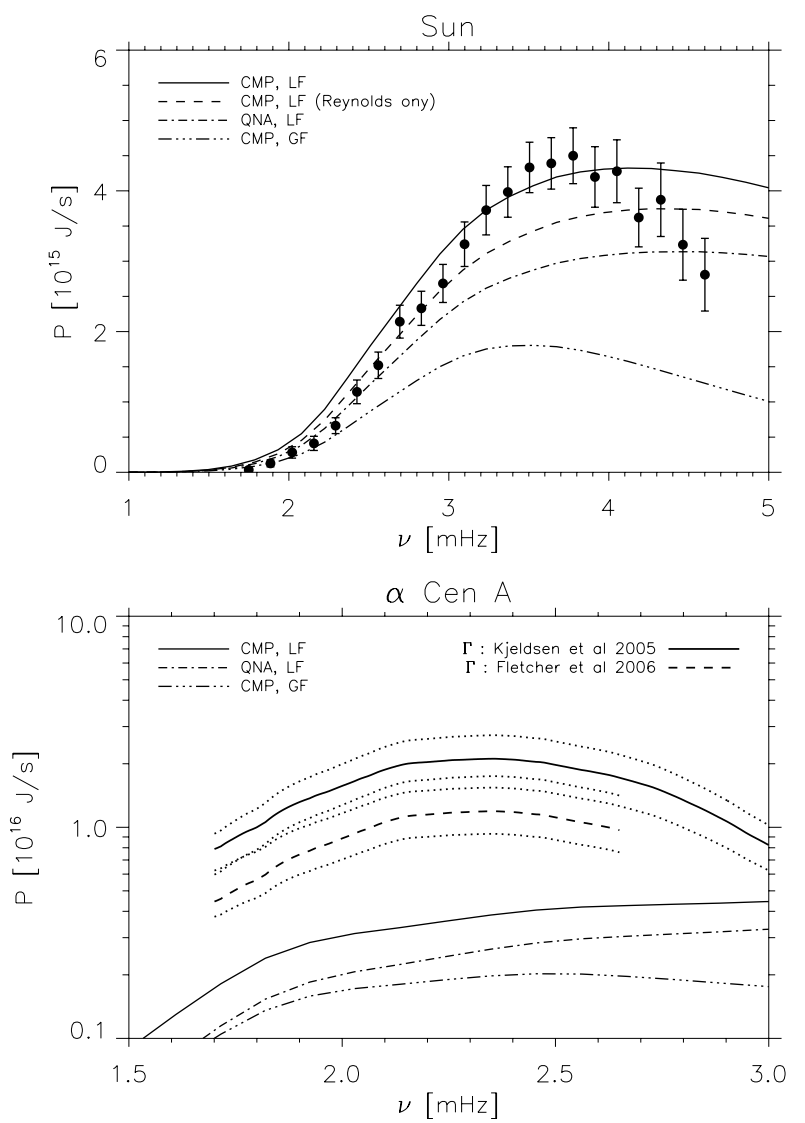

Figure 2. Top: Rates at which energy is injected into the solar modes. The filled circles correspond to the helioseismic constraints obtained by Baudin et al. (2005). The lines correspond to different theoretical calculations: the solid line uses the Lorentzian function (LF) and the CMP, the dashed line is as the solid line with only the contribution of the Reynolds stress, the dot-dashed line uses the LF and the QNA closure model, the tripledot-dashed line uses the Gaussian function (GF) and the CMP. Bottom: Same as top for $\alpha$ Cen A. The thick lines correspond to the constraints obtained from the observed spectrum derived by Kjeldsen et al. (2005) and the averaged mode line-widths derived by Kjeldsen et al. (2005, the thick solid line) and by Fletcher et al. (2006, the thick dashed line). The thick dotted lines correspond to extreme values derived from the estimated errors on the amplitude and line-width measurements. The thin lines have the same meaning as in the top panel.

in terms of mode excitation rates. This requires the mode masses $\left(\mathcal{M}=I / \xi_{\mathrm{r}}^{2}\right)$, which are, as in Samadi et al. (2004), evaluated at the photosphere.

\section{Conclusion}

Comparison between recent helioseismic constraints obtained by Baudin et al. (2005) and the theoretical calculations based on the quasi-normal approximation (QNA) confirms the result of SNS03 that the Lorentzian function (LF) results in a better agreement with the observations than the Gaussian function (GF).

As for the Sun, we find that for $\alpha$ Cen Athe theoretical calculations based on a Lorentzian eddy-time correlation function is closer to the observations than those based on a Gaussian one.

In the case of the Sun, B06b have shown that the QNA results in a significant underestimation of the contribution of Reynolds stress to the mode excitation. On the other hand, the so-called closure model with plumes (CMP) proposed by B06a increases significantly this contribution. The theoretical calculations limited to the Reynolds stress only underestimates by $\sim 15 \%$ the maximum in the excitation rates $P$ derived by Baudin et al. (2005). On the other hand, when the entropy contribution is added, the theoretical calculations based on this improved closure model fit the helioseismic data.

In the case of $\alpha$ Cen $\mathrm{A}$, the theoretical calculations underestimate the observations by a factor ten. We note also that the accuracy at which mode amplitudes and line-widths 
are measured is more or less of the same order as the difference between theoretical calculations using the QNA and those using the CMP. This emphasises the need for more accurate seismic data.

Whatever the assumptions about the eddy-time correlation or the closure model, the theoretical calculations are found to be closer to the constraints derived using the linewidths of Fletcher et al. (2006) than to those obtained for Kjeldsen et al. (2005)'s.

The space based mission CoRoT (see the recent review by Michel et al. 2006) is the only asteroseismology mission that in the very near future will enable us to derive, for a large set of solar-like oscillating stars with different effective temperature and gravity, the rates at which energy is supplied to the modes by turbulent convection. The quality of the data is expected to be significantly higher than current observations. Hence, these data will thus very likely permit to discriminate between the best description for the eddy-time correlation function and closure models.

\section{Discussion}

For the Sun, the largest discrepancies between theoretical calculations and the observations are seen at high frequency $(\nu \gtrsim 4 \mathrm{mHz})$ and at low frequency $(\nu \lesssim 3 \mathrm{mHz})$.

Part of the remaining discrepancies with the helioseismic data can be attributed to the closure models considered here which are not able to reproduce correctly the FOM from the 3D simulation in the super-adiabatic region. Indeed, as seen in Figure 2.1, all the closure models considered here overestimate the FOM. B06b have shown that the main effect on the excitation rates is at rather high frequency and results in an increase of the order of $\sim 15 \%$ when the CMP is adopted.

In B06b, the one-point correlation of the velocity was generalized to a two-point correlation product using the QNA (see $\S 2.2$ ). It was shown in B06b that, at small scale length, this approximation reproduces satisfactorily the constraints derived from the $3 \mathrm{D}$ simulation. At large scale length, however, this approximation overestimates those constraints. The effect on the excitation rates of this overestimation at large scale remains to be evaluated.

Finally, we must point out that the calculation of the mode excitation rates requires the calculation of the mode eigenfunctions. The latter are obtained on the basis of global $1 \mathrm{D}$ stellar models. In such stellar models, the structure of the outer layer is very poorly modeled. Therefore, this poor description must have some (currently unknown) consequences on the inferred properties of the mode eigenfunctions in particular on the high frequency modes which are almost confined near the photosphere. In turn, as most of the excitation occurs near the photosphere, this poor description is likely to introduce some (unknown) biases on the predicted excitation rates in particular at high frequency.

The discrepancies at low frequency can probably be attributed to the adopted model for the eddy time-correlation which is probably too simple. Indeed, the departures from a Gaussian behavior were attributed in SNS03 to the plumes, which are more turbulent than the granules. Near the photosphere, the excitation of the p modes is almost solely due to the plumes. However, one Mm deeper, the excitation is no longer dominated by the plumes. As the granules are less turbulent than the plumes, their time-correlations follow more likely a GF distribution than a LF. Then one Mm deeper the eddy-time correlation is expected to lie between the LF and the GF. This however remains to be checked.

For $\alpha$ Cen A, the large discrepancies between the observations and the theoretical calculations remain to be understood. In addition to what is mentioned above for the case of the Sun, the ways the mode excitation rates were computed in the particular case 
of $\alpha$ Cen Amay have some (unknown) effects on the computed excitation rates. Indeed, the adiabatic eigenfunctions were obtained from a 1D model based on the local mixinglength theory. Finally, the 3D simulation considered here uses solar abundances. On the observational side, we shall mention that the excitation rates were derived from the observations using mode masses evaluated arbitrarily at the photosphere. As the doppler measurements used a lot of spectral lines, it is not obvious to evaluate the observational mode mass.

The work performed in SNS03 for the case of the Sun has been extended by Samadi et al. (2005) to the case of stars lying on the main sequence for which solar-like oscillations are expected. They have found that the maximum in the mode amplitudes, $V_{\max }$, scales as $(L / M)^{s}$ where $s$ is the slope of the scaling law, $L$ is the luminosity and $M$ is the mass of the star. The slope $s$ was found to be significantly sensitive to the adopted eddy-time correlation function. As for the Sun and $\alpha$ Cen A, theoretical calculations assuming an LF fits best the maximum oscillation amplitudes detected for a set of $\sim 10$ stars including main-sequence stars as well as some red giant stars. However, these calculations rely on the QNA. A preliminary work tends to show that the asymmetry between the updrafts and the downdrafts does not change significantly between the few $3 \mathrm{D}$ simulations of main-sequence stars investigated in this work and that the CMP remains valid according to these 3D simulations. Hence, we expect that the difference between the effect on the excitation rates of the CMP model and that of the QNA remains constant for intermediate massive stars lying on the main sequence. This however needs to be confirmed using more $3 \mathrm{D}$ simulations and extended to other domains of the HR diagram.

\section{Acknowledgements}

FK is grateful to V.M. Gryanik and J. Hartmann for discussions on their model and their observational data. We thank $\AA$. Nordlund and R. F. Stein for making their code available to us. Their code was made at the National Center for Supercomputer Applications and Michigan State University and supported by grants from NASA and NSF.

\section{References}

Abdella, K. \& McFarlane. 1997, J. Atmos. Sci. 54, 1850

Balmforth, N. J. 1992, MNRAS 255, 639

Baudin, F., Samadi, R., Goupil, M.-J., et al. 2005, A\& A 433, 349

Bedding, T. R. \& Kjeldsen, H. 2006, Memorie della Societa Astronomica Italiana 77, 384

Bedding, T. R., Kjeldsen, H., Butler, R. P., et al. 2004, ApJ 614, 380

Belkacem, K., Samadi, R., Goupil, M. \& Kupka, F. 2006a, A 6 A 460, 173

Belkacem, K., Samadi, R., Goupil, M. \& Kupka, F. 2006b, A\& A 460, 183

Belkacem, K., Samadi, R., Goupil, M., Kupka, F. \& Baudin, F. 2006c, in SF2A-2006: Semaine de l'Astrophysique Francaise, meeting held in Paris, France, June 26-30, 2006, Eds.: D. Barret, F. Casoli, T. Contini, G. Lagache, A. Lecavelier, and L. Pagani, Vol. in press

Chaplin, W. J., Elsworth, Y., Isaak, G. R., et al. 1998, MNRAS 298, L7

Chaplin, W. J., Houdek, G., Elsworth, Y., et al. 2005, MNRAS 360, 859

Fletcher, S. T., Chaplin, W. J., Elsworth, Y., Schou, J. \& Buzasi, D. 2006, MNRAS 371, 935

Goldreich, P. \& Keeley, D. A. 1977, ApJ 212, 243

Gough, D. O. 1977, ApJ 214, 196

Gryanik, V. \& Hartmann, J. 2002, J. Atmos. Sci. 59, 2729

Kjeldsen, H., Bedding, T. R., Butler, R. P., et al. 2005, ApJ 635, 1281

Kupka, F. \& Robinson, F. 2007, MNRAS 374, 305

Lesieur, M. 1997, Turbulence in fluids (Kluwer Academic Publishers)

Michel, E., Samadi, R., Baudin, F., Auvergne, M. \& the Corot Team. 2006, Memorie della Societa Astronomica Italiana 77, 539 
Miglio, A. \& Montalbán, J. 2005, A\&A 441, 615

Rieutord, M. \& Zahn, J.-P. 1995, A\&̈A 296, 127

Samadi, R. \& Goupil, M. . 2001, A\&A 370, 136

Samadi, R., Goupil, M.-J., Alecian, E., et al. 2005, JapA 26, 171

Samadi, R., Goupil, M. J., Baudin, F., et al. 2004, in Proceedings of the SOHO 14/GONG 2004 Workshop (ESA SP-559). "Helio- and Asteroseismology: Towards a Golden Future". Editor: D. Danesy., 615 (astro-ph/0409325)

Samadi, R., Nordlund, Å, Stein, R. F., Goupil, M. J. \& Roxburgh, I. 2003a, A\&̊A 404, 1129

Samadi, R., Nordlund, Å., Stein, R. F., Goupil, M. J. \& Roxburgh, I. 2003b, A\&\&A 403, 303

Schou, J. \& Buzasi, D. L. 2001, in ESA SP-464: SOHO 10/GONG 2000 Workshop: Helio- and Asteroseismology at the Dawn of the Millennium, ed. A. Wilson \& P. L. Pallé, 391-394

Stein, R. F. \& Nordlund, A. 1998, ApJ 499, 914

\section{Discussion}

V.M. Canuto: How do you determine the filling factor?

R. SAmADi: This quantity is obtained directly from the 3D simulation.

Tim BedDing: Comment: It is very nice that you can compare your models with our observations. But please do not compare with amplitudes of individual peaks. There is a systematic effect because those peaks are selected because they happen to be highest during the observations. (This is true even if you average over many peaks). Instead, please use the amplitudes we estimated by smoothing and then noise-correcting the power spectrum. For $\alpha$ Cen A and B, this is shown in Kjeldsen et al. (2005).

R. SAmadi: Yes, I agree with you, the comparison should be done using the type of amplitude spectrum you mention. (Thanks to T. Bedding this is now done in the present proceedings).

R.F. Stein: First, we have compared the non-adiabatic eigenfunctions from our simulations with the eigenfunctions of J. Christensen-Dalsgaard and they agree very closely, so I don't think that using non-adiabatic eigenfunctions is important. Second, you add the absolute values squared of the turbulent pressure and entropy fluctuation contributions. In fact, they do not always add but sometimes cancel each other. Hence, adding the contributions linearly would be more accurate.

R. SAMADI: We have compared the non-adiabatic eigenfunctions computed using the time-dependent formalism of Gabriel for convection (see Grigahcene et al. 2005) with adiabatic eigenfunctions and found important differences. Hence, different treatments of convection affect differently the eigenfunctions. Further work is thus needed on that issue. Concerning your remark about the turbulent pressure and entropy fluctuation, within the theoretical framework of our formalism, the crossing term between the Reynolds stress source term and the entropy source term vanishes on average. Hence, further theoretical work is required in order to correctly model this crossing term.

J. Christensen-DalsgandD: Comment: The computations of damping rates (needed to determine the amplitude) is probably even more uncertain than the computation of the energy input. For the red giant $\xi$ Hya there is a striking discrepancy between observed and computed damping rates. Better understanding, and better observations, are needed. 